\title{
MicroRNA-205 Regulates the Calcification and Osteoblastic Differentiation of Vascular Smooth Muscle Cells
}

\author{
Weiwei Qiao ${ }^{a, b} \quad$ Li Chen $^{a, c} \quad$ Mingxiang Zhang ${ }^{a}$ \\ The Key Laboratory of Cardiovascular Remodeling and Function Research, Chinese Ministry of \\ Education and Chinese Ministry of Public Health, Qilu Hospital, Shandong University, Jinan, Shandong, \\ China; ${ }^{b}$ Department of Cardiology, Yantaishan Hospital, Yantai, Shandong, China; 'Department of \\ Geriatrics, Anhui Provincial Hospital Affiliated to Anhui Medical University, Hefei, Anhui, China
}

\section{Key Words}

Calcification • Vascular smooth muscle cells $\bullet \operatorname{miR}-205 \cdot \operatorname{Runx2} \cdot$ Smad1

\begin{abstract}
Objectives: We investigated the role of miR-205 in the osteogenic differentiation of vascular smooth muscle cells (VSMCs). Methods: Osteogenic differentiation of human aortic smooth muscle cells (HASMCs) was induced by $10 \mathrm{mM} \beta$-glycerophosphate $(\beta-G P)$. Alizarin Red $S$ staining, alkaline phosphatase (ALP) activity and osteocalcin secretion were used to determine osteogenic differentiation of HASMCs. Quantitative reverse transcriptase polymerase chain reaction (qRT-PCR) was used to measure the expression of miR-205 in HASMCs. Results: The expression of endogenous miR-205 was decreased in HASMCs during $\beta$-glycerophosphateinduced calcification. Overexpression of miR-205 inhibited the differentiation of HASMCs into osteoblast-like cells, as evidenced by a decrease in ALP activity, osteocalcin secretion, and Runx2 expression, whereas miR-205 depletion enhanced osteoblastic differentiation of HASMCs. Runx2 and Smad1 were identified as direct targets of miR-205 by computational analysis and experimental assays. Conclusion: The present study shows that miR-205 may negatively regulate the $\beta$-glycerophosphate-induced calcification of HASMCs, at least partially by targeting Runx2 and Smad1.
\end{abstract}




\section{Introduction}

Vascular calcification is the deposition of calcium phosphate crystals in cardiovascular tissues [1]. It reduces vessel elasticity, resulting in impaired cardiovascular hemodynamics and increased morbidity and mortality associated with hypertension, aortic stenosis, cardiac hypertrophy, and other cardiovascular diseases [2]. In the past, vascular calcification was considered a passive and degenerative process; however, recent research has shown that this process is regulated by a mechanism similar to that of bone formation [3]. The identification of bone calcification regulatory factors in blood vessels and the differential expression of these factors in nondiseased and diseased vessels verified that vascular calcification is a regulated process analogous to skeletal bone formation but orchestrated by vascular smooth muscle cells (VSMCs) [4-6]. VSMCs release matrix vesicles with the capacity to concentrate calcium and phosphate, acting as nidus for mineralization, and undergo a phenotypic transition in which the expression of transcription factors associated with differentiated chondrocytes and osteoblasts induce the expression of mineralization regulating proteins such as alkaline phosphatase, osteopontin and osteocalcin [7].

MicroRNAs (miRNAs) are a class of small (16-25 nucleotides), single-stranded noncoding RNAs that negatively regulate gene expression through incomplete base-pairing to the $3^{\prime}$ untranslated region (3'-UTR) of target mRNAs, inducing mRNA degradation or blocking translation $[8,9]$. miRNAs have been studied extensively because of their roles as regulators of cell differentiation, growth, proliferation and apoptosis, and therefore their involvement in several diseases, in particular their role in cancer [10]. Importantly, miRNAs are highly expressed in the cardiovascular system and their involvement not only in cardiovascular development, but also in cardiovascular diseases including atherosclerosis and pulmonary arterial hypertension has only recently began to be understood $[11,12]$. Several miRNAs, such as miR-125b, miR-204, miR-29a/b, miR-30b/c and miR-133a, have been identified that are involved in VSMC calcification [13-17]. However, the mechanisms underlying the effect of miRNAs and their targets in the modulation of VSMC differentiation leading to vascular calcification remain to be elucidated.

MiR-205 was previously shown to be among a series of miRNAs that regulate osteoblast differentiation and bone formation by directly targeting Runx2 [18, 19]. Whether miR-205 is also involved in VSMC osteogenic differentiation has not yet been explored. In this study, we found that miR-205 is a negative regulator of osteogenic differentiation in VSMCs, and identified Runx2 and Smad1 as its direct targets.

\section{Materials and Methods}

\section{Cell culture}

Human aortic smooth muscle cells (HASMCs) were purchased from Promocell (Heidelberg, Germany) and cultured in DMEM supplemented with 10\% fetal bovine serum (FBS) and 100 units/ml penicillin/ streptomycin at $37^{\circ} \mathrm{C}$ and $5 \% \mathrm{CO}_{2}$. To induce calcification, the cells were incubated in DMEM containing $15 \%$ FBS supplemented with $10 \mathrm{mM} \beta$-glycerophosphate $(\beta-\mathrm{GP})$.

\section{Measurements of ALP activity and osteocalcin secretion}

To determine the activity of alkaline phosphatase (ALP), cells were washed with PBS and scraped into

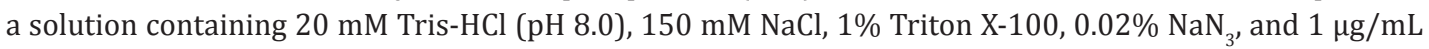
aprotinin. Lysates were homogenized and assayed for ALP activity by measuring the release of p-nitrophenol at $37^{\circ} \mathrm{C}$ using a spectrophotometer. Results were normalized to total cellular protein content. Osteocalcin secretion into the culture media was measured by using a specific radioimmunoassay kit (DiaSorin Corp., Stillwater, MN, USA) according to the manufacturer's instructions. Protein expression was normalized to total cellular protein measured by the Bradford protein assay. 


\section{Alizarin Red S staining}

The formation of a mineralized matrix was determined by Alizarin Red S staining. HASMCs cultured in DMEM supplemented with $10 \mathrm{mM} \beta$-GP were fixed in $70 \%$ ethanol for $1 \mathrm{~h}$ at room temperature and stained with $40 \mathrm{mM}$ Alizarin Red S for $10 \mathrm{~min}$. Then, cells were washed with PBS to eliminate nonspecific staining and the stained matrix was photographed using a digital microscope. For quantification of staining, the Alizarin Red S stain was released from the cell matrix by incubation in cetyl-pyridinium chloride for $15 \mathrm{~min}$ and the amount of released dye was measured by spectrophotometry at $540 \mathrm{~nm}$. The results were normalized to total cellular protein content.

\section{Quantitative reverse transcriptase PCR (qRT-PCR)}

qRT-PCR was performed as described previously [20]. Briefly, total RNA was isolated using TRIzol reagent (Invitrogen). For quantification of miR-205, reverse transcription and qPCR were performed using the TaqMan MicroRNA Reverse Transcription Kit and TaqMan miRNA assay (Applied Biosystems, Foster City, CA, USA). qRT-PCR analyses for RUNX2 and Smad1 mRNAs were performed by using with SuperScript II (Invitrogen) and SYBR Green PCR Maser Mix (Applied Biosystems). Experiments were performed in triplicate. Relative expression of miRNA or mRNA was normalized to the expression of U6 or $\beta$-actin and evaluated by the $2^{-\Delta \Delta C t}$ method.

\section{Western blotting}

Cells were harvested and lysed in RIPA buffer with protease and phosphatase inhibitors (Roche, Indianapolis, IN, USA). The protein concentration was assayed by BCA (Bio-Rad). Approximately 20 to $50 \mu \mathrm{g}$ of total protein was separated by $10 \%$ SDS-PAGE and transferred to polyvinylidene difluoride membranes. Membranes were blocked in tris-buffered saline containing 0.05\% Tween 20 and 5\% skim milk and then probed with antibodies against Runx2, Smad1 and $\beta$-actin (Santa Cruz Biotechnology, Santa Cruz, California, USA). Membranes were then washed and incubated with the appropriate secondary antibodies and detected using enhanced chemiluminescence. Band intensity was quantified by using ImageJ software.

\section{Plasmid construction}

The full-length open reading frame of Runx2 or Smad1 was cloned into pcDNA3.1 (+) to generate their expression vectors. The wild-type Runx2 or Smad1 3'-UTR (WT) was cloned into the pGL3-basic vector (Promega, Madison, WI, USA). Site-directed mutagenesis of the miR-205 seed sequence in the $3^{\prime}$-UTR (Mut) was performed using the QuikChange ${ }^{\mathrm{TM}}$ Site-Directed Mutagenesis Kit (Stratagene, La Jolla, CA, USA).

\section{Oligonucleotide transfection}

Cells were seeded into 6-well plates, transfected with miR-205 mimics, anti-miR-205, Runx2 siRNA, Smad1 siRNA or the respective controls (GenePharma, Shanghai, China) using Lipofactamine ${ }^{\mathrm{TM}}$ RNAiMAX (Invitrogen), or transfected with plasmids using Lipofactamine 2000 reagent (Invitrogen). The cells were collected for assays $48 \mathrm{~h}$ after transfection.

\section{Luciferase assays}

For luciferase assays, HASMCs were cultured in 24-well plates and co-transfected with luciferase reporter plasmid and miR-205 mimics and pRL-TK vector (Promega) using Lipofectamine 2000 (Invitrogen). Cells were harvested and lysed $48 \mathrm{~h}$ after transfection, and luciferase activity was measured using the Dual-Luciferase Reporter Assay System (Promega). Renilla-luciferase was used for normalization. The experiments were performed independently in triplicate.

\section{Statistical analysis}

Data are expressed as mean \pm SD from at least three independent experiments. Statistical analysis was performed using Student's $t$ test. $P<0.05$ was considered statistically significant. 


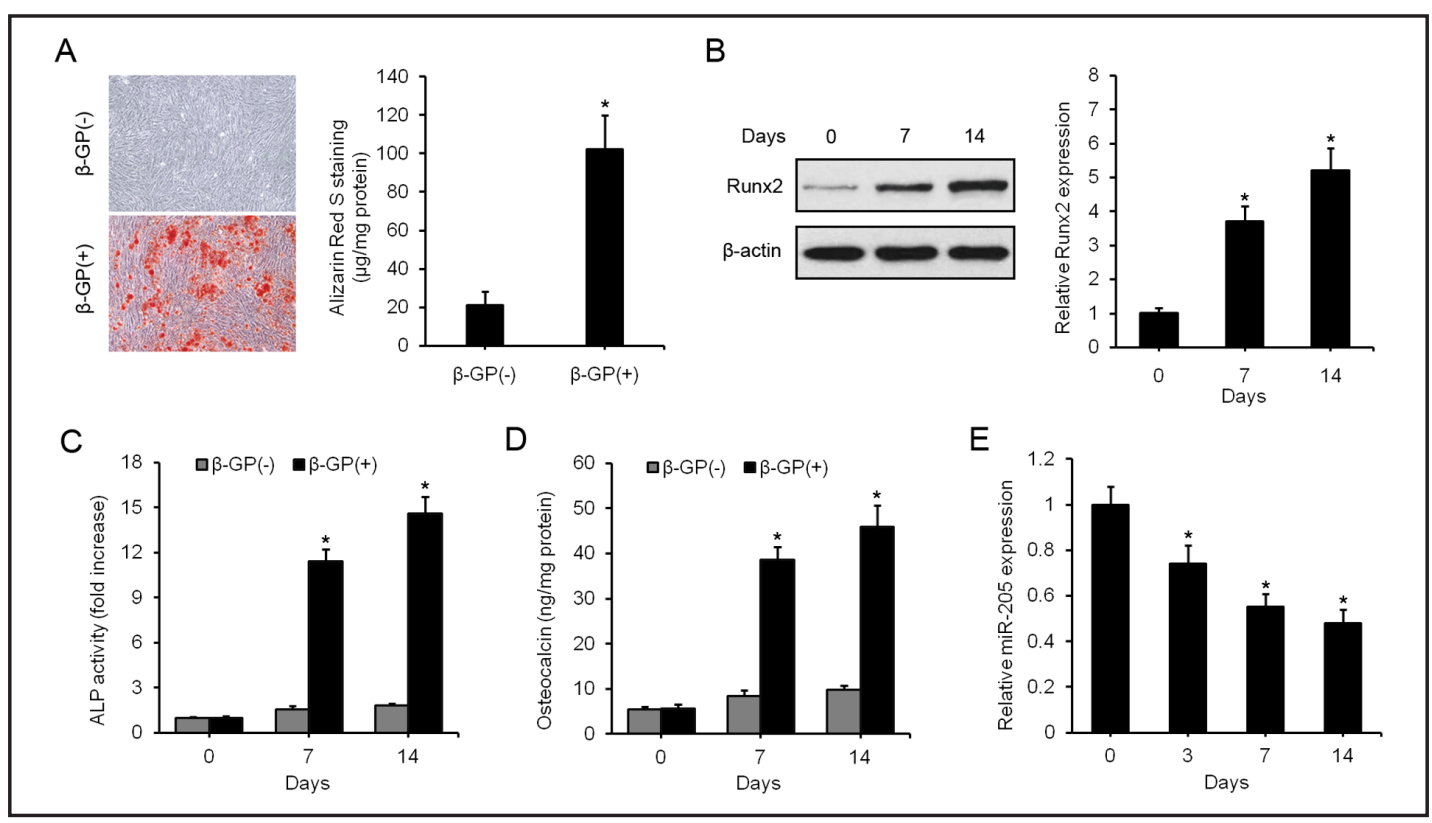

Fig. 1. miR-205 was down-regulated during the calcification of HASMCs. HASMCs were treated with or without $10 \mathrm{mM} \beta$-glycerophosphate ( $\beta$-GP) for 14 days to induce osteoblastic differentiation. (A) Alizarin Red S staining was performed on day 14. Representative microscopic images are shown on the left; quantification of Alizarin Red S staining is shown on the right. (B) The expression of Runx2 was analyzed by western blotting with $\beta$-actin as the loading control. (C) ALP activity was measured during the course of differentiation. (D) Osteocalcin level was determined by enzyme-linked immunosorbent assay. (E) Relative expression of miR-205 was measured by qRT-PCR. ${ }^{*} P<0.05$ versus non-induced group.

\section{Results}

miR-205 levels are reduced in HASMCS undergoing osteoblastic differentiation

To induce the calcification of HASMCs, cells were incubated in DMEM supplemented with $10 \mathrm{mM} \beta$-GP. As shown in Fig. 1A-D, increased matrix mineralization, Runx2 expression, ALP activity and osteocalcin levels were induced when cells were cultured under calcification conditions. To examine the possible association between miR-205 and HASMC calcification, the expression levels of miR-205 were determined by qRT-PCR, which showed a statistically significant downregulation of the expression of miR-205 in response to $\beta$-GP treatment (Fig. $1 \mathrm{E})$, indicating that miR-205 is downregulated during the calcification of HASMCs.

\section{miR-205 inhibits $\beta$-GP-induced osteoblastic differentiation of HASMCs}

To further investigate the role of miR-205 in the osteoblastic differentiation of HASMCs, cells were transfected with miR-205 mimics or anti-miR-205 or the respective controls, and the levels of miR-205 were determined by qRT-PCR. miR-205 up- and down-regulation could be maintained for approximately 8 days in these cells (Fig. 2A). miR-205 silencing significantly enhanced osteoblastic differentiation, which was indicated by enhanced in vitro matrix mineralization, up-regulated Runx2 protein expression, increased ALP activity and osteocalcin secretion in anti-miR-205-transfected HASMCs compared with cells transfected with miR-ctrl (Fig. 2B-E). In contrast, matrix mineralization, Runx2 protein expression, ALP activity and osteocalcin secretion were reduced in miR-205 minics-treated HASMCs (Fig. 2B-E). Together, these results indicate that miR-205 plays a negative role in the regulation of osteoblastic differentiation of HASMCs.

Smad1 and Runx2 are common targets of miR-205

Given that Runx2 and Smad1, which are key downstream mediators of bone morphogenetic protein-2 signaling [21], have been previously reported to be target genes of 


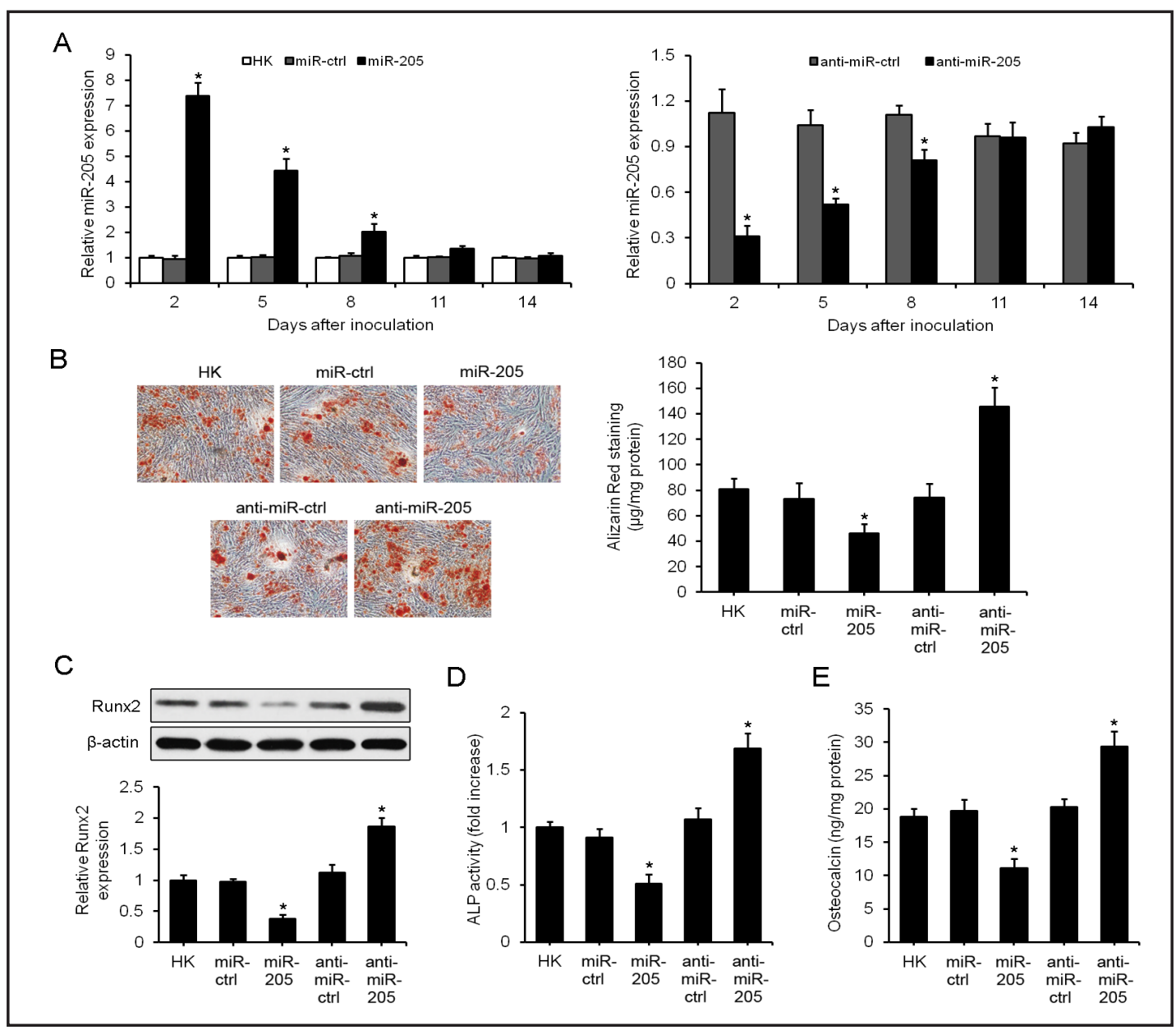

Fig. 2. miR-205 inhibits $\beta$-GP-induced osteoblastic differentiation of HASMCs. (A) qRT-PCR analysis of miR-205 levels in HASMCs transfected with miR-205 mimics or anti-miR-205 or the respective controls (Control). (B) Alizarin Red S staining. (C) Western blotting analysis of the Runx2 expression in the transfected HASMCs treated with $10 \mathrm{mM} \beta$-GP for 72 hours. (D) ALP activity was determined by ALP assay and expressed relative to total cellular protein. (E) Osteocalcin levels were measured by ELISA and expressed relative to total cellular protein. ${ }^{*} P<0.05$ versus control group. HK, housekeeping (control).

miR-205 [18, 22], we next aimed to determine whether they are involved in the regulation of osteoblastic differentiation of HASMCs. Fig. 3A shows the putative target sites for miR-205 in the 3'-UTRs of Runx2 and Smad1. To analyze the relationship between miR-205 and Runx2 and Smad1, HASMCs were co-transfected with miR-205 mimics and luciferase reporter constructs containing the wild-type (wt) or mutant (mut) miR-205 target sites in the Runx2 or Smad1 3 '-UTR, and luciferase activities were measured $48 \mathrm{~h}$ after transfection. The results showed that overexpression of miR-205 significantly decreased the luciferase activity of the wt-3'-UTRs of Runx2 and Smad1 compared to miR-control transfected cells whereas it had no effect on the mut-3'-UTRs (Fig. 3B), indicating that miR-205 suppressed the expression of Runx2 and Smad1 by directly binding to target sites in their $3^{\prime}$-UTRs. Furthermore, qRT-PCR analysis showed that miR-205 overexpression had no significant effect on Runx2 and Smad1 mRNA levels (Fig. 3C). However, miR-205 overexpression significantly downregulated the expression of the Runx2 and Smad1 proteins compared to untransfected controls or miRcontrol transfected cells (Fig. 3D). These results support the bioinformatics predictions indicating Runx2 and Smad1 3'-UTRs as direct targets of miR-205. 


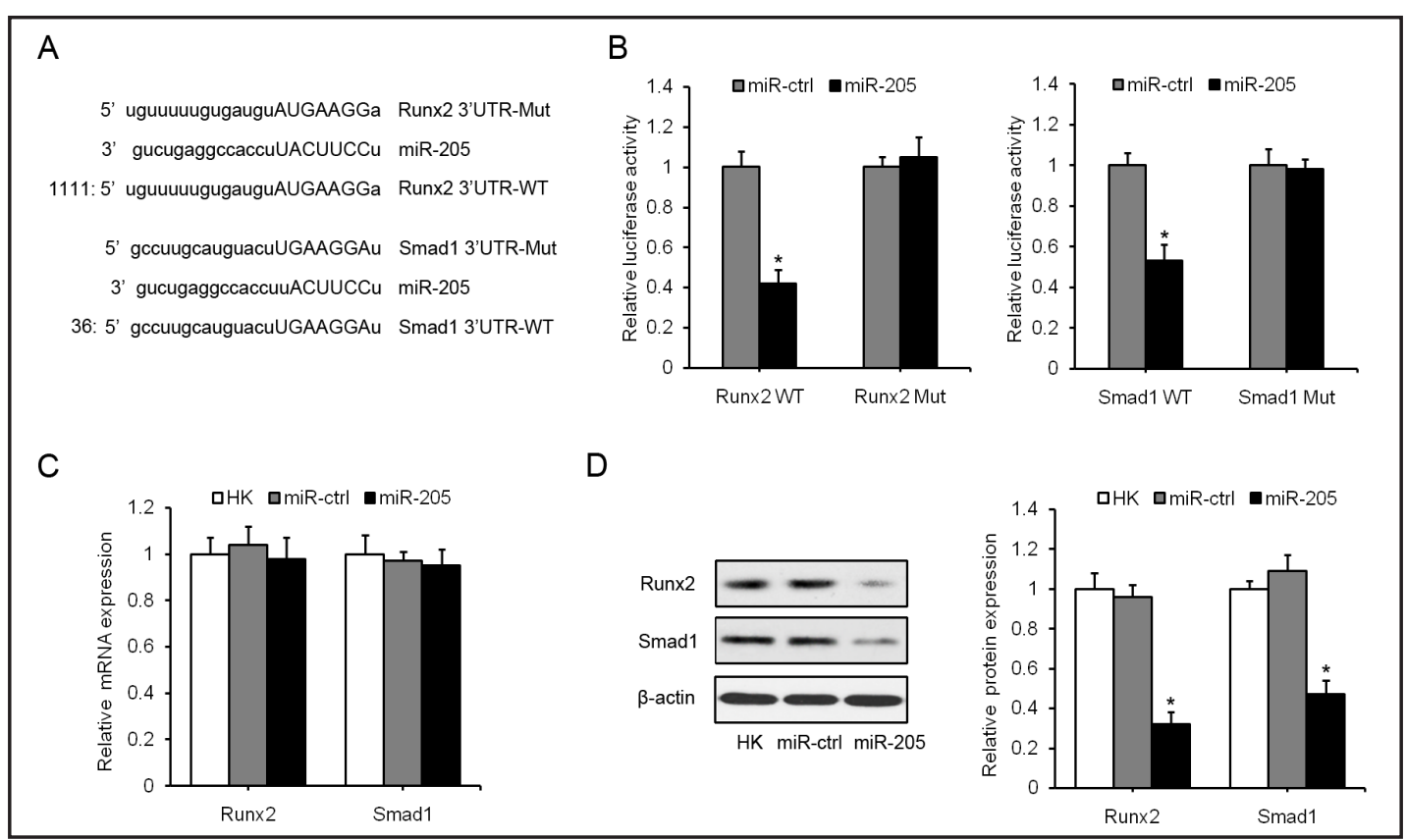

Fig. 3. Runx2 and Smad1 are targets of miR-205. (A) Schematic illustration of the predicted miR-205-binding sites in the 3'-UTRs of Runx2 and Smad1. (B) Luciferase reporter assays. HASMCs were co-transfected with luciferase reporter plasmids containing the wild-type (WT) or mutant (Mut) miR-205 target sites in the Runx2 or Smad1 3'-UTRs and miR-205 or miR-ctrl precursors. Luciferase activities were measured 48 hours post transfection and normalized to Renilla luciferase activity. (C) Runx2 and Smad1 mRNA levels were analyzed upon miR-205 or miR-ctrl transfection by qRT-PCR. (D) Runx2 and Smad1 protein levels were analyzed by western blotting in cells transfected with miR-205 or miR-ctrl with $\beta$-actin as the loading control. ${ }^{*} P<0.05$ versus miR-ctrl group. HK, housekeeping (control).

Runx2 and Smad1 mediate miR-205-regulated osteoblastic differentiation of HASMCS

We further explored whether Runx2 and Smad1 deregulations were required for the suppressive effect of miR-205 in regulating the osteogenic differentiation of HASMCs. We restored the expression of Runx2 and Smad1 in miR-205-overexpressing HASMCs by transfection of Runx2 and Smad1 ORF constructs without 3'-UTRs (Fig. 4A), and assessed their effect on ALP activity and osteocalcin levels. As shown in Fig. 4B, overexpression of Runx2 or Smad1 significantly increased ALP activity and osteocalcin secretion, suggesting that Runx2 or Smad1overexpression impaired the effect of miR-205 during osteoblastic differentiation of HASMCs (Fig. 4B). Furthermore, cotransfection of Runx2 siRNA or Smad1 siRNA with anti-miR-205 almost completely blocked the positive role of anti-miR-205 on these parameters (Fig. 4C). Taken together, these results suggested that Runx2 and Smad1 are functional targets of miR-205.

\section{Discussion}

Vascular calcification is a complex pathobiological process and despite its importance in the pathogenesis of various cardiovascular diseases, the underlying mechanisms are just recently beginning to be understood. Vascular calcification has traditionally been divided into intimal calcification, resulting from the osteoblastic differentiation of smooth muscle cells in the neointima, and medial calcification, which results from the differentiation of endothelial cells or VSMCs into bone-like cells and causes arterial stiffening, hypertension and left ventricular hypertrophy $[23,24]$. Coronary artery calcification is an independent predictor 


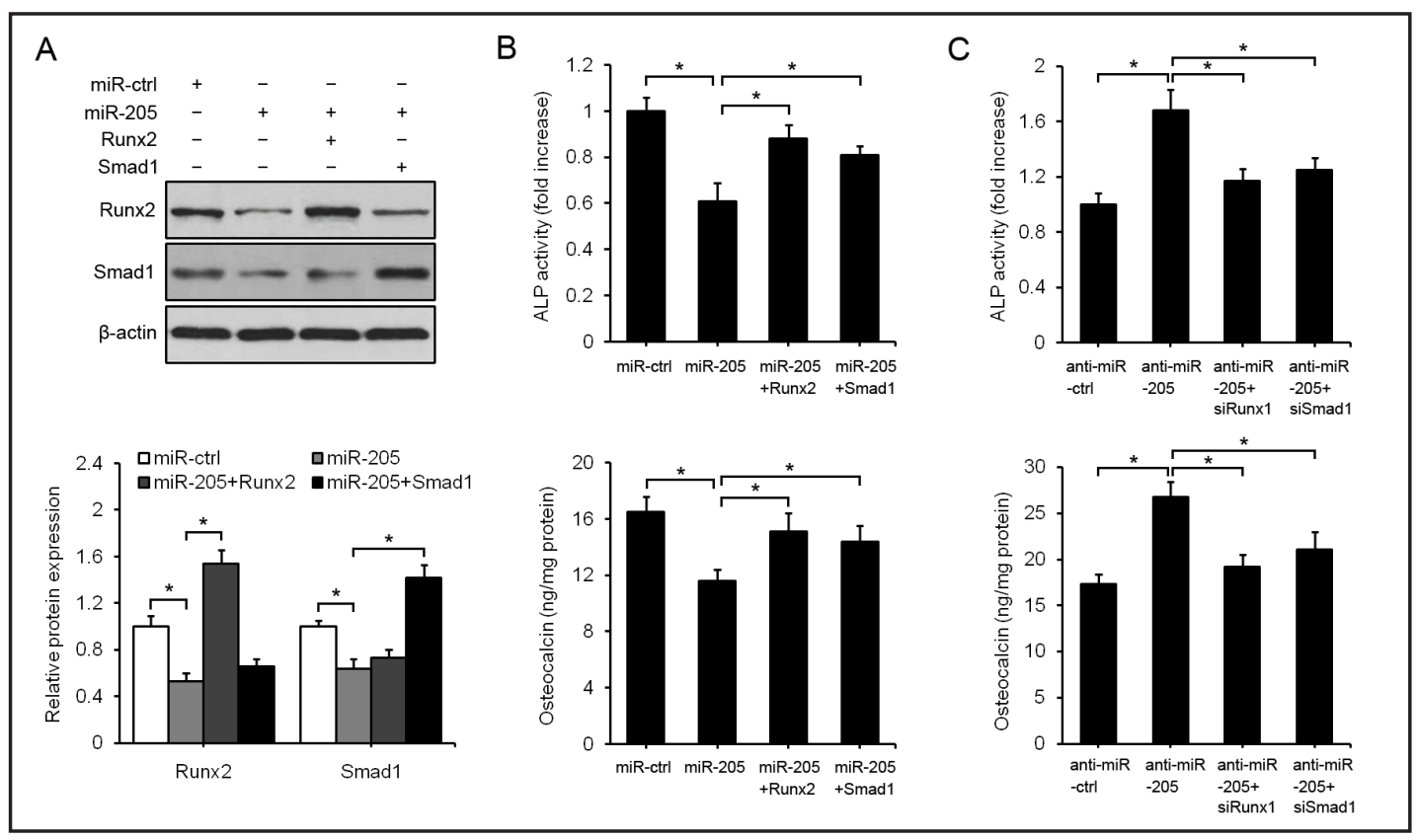

Fig. 4. Runx2 and Smad1 mediate miR-205-regulated osteoblastic differentiation of HASMCs. HASMCs were transfected with the indicated miR-205 and/or the indicated cDNAs, and treated with $10 \mathrm{mM} \beta$-GP for 3 days. The protein levels of Runx2 and Smad1 were measured by western blotting (A). ALP activity and osteocalcin levels were measured (B). (C) HASMCs were transfected with anti-miR-205 and/or the indicated siRNAs and treated with $10 \mathrm{mM} \beta$-GP. Osteogenic differentiation was determined by ALP activity and osteocalcin secretion after 3 days of osteogenic induction. ${ }^{*} P<0.05$.

of cardiovascular disease and mortality [25], and calcification of the abdominal aorta is an important predictor of subsequent cardiovascular events [26]. Therefore, elucidation of the mechanisms underlying the process of vascular calcification and identification of the factors involved is important. In the present study, we showed that miR-205 negatively regulates the conversion of VSMCs into bone-like cells and identified Runx2 and Smad1 as direct targets of miR-205, whose miR-205 induced downregulation mediates its effect on osteogenic differentiation.

Although several miRNAs that are expressed in VSMCs have been identified [1317], investigation of their roles in vascular calcification is limited. Recently, miR-205 was identified as a miRNA targeting the osteogenic regulator Runx2 in mesenchymal cell types $[18,19]$. However, it is unknown if miR-205 exerts any effect on calcification of VSMCs. In the present study, we found that miR-205 was downregulated during HASMC calcification and inhibits the $\beta$-GP-induced osteoblastic differentiation of HASMCs. Gain of function studies showed that overexpression of miR-205 decreased ALP activity, osteocalcin secretion and the expression of Runx2, whereas inhibition of miR-205 expression had the opposite effects. This inverse correlation between miR-205 expression and the activity and levels of important markers of osteoblast differentiation demonstrated the role of miR-205 as a negative regulator of osteogenic differentiation.

To further investigate the molecular mechanism by which miR-205 inhibits osteogenic differentiation, we conducted computational search for the target genes of miR-205 and found that Smad1 was a possible target in addition of Runx2, because complementary sequence of miR-205 is identified in the $3^{\prime}$-UTR of their mRNAs. Our results showed that miR-205 overexpression downregulated Runx2 and Smad1 protein, and luciferase activity assays confirmed the direct interaction between miR-205 and the 3'-UTRs of Runx2 and Smad1. 
Runx2 is an essential transcription factor for osteoblast differentiation. It also plays important roles in the osteogenic transdifferentiation as well as in the calcification of VSMCs [14]. Smad1 is phosphorylated in response to the binding of bone morphogenetic protein (BMP) to BMP receptors on the cell surface and forms a complex with other Smad proteins that is translocated to the nucleus, where it regulates the transcription of BMP target genes [27]. BMP-Smad signaling controls osteoblast differentiation and bone formation. Furthermore, BMP activated Smads interact with Runx2 to enhance the transcription of osteoblast-specific genes [28], and the interaction of Smad1 with Runx2 on the promoter of target genes controls osteoblast gene expression and differentiation [29]. The regulation of Runx2 and Smad1 by different miRNAs in relation to osteogenic differentiation has been shown in previous studies $[14,16,17,30,31]$. In the present study, we found that overexpression of Runx2 or Smad1 reversed the negative effects of miR-205 on ALP activity and osteocalcin secretion, whereas silencing of Runx2 or Smad1 abolished the anti-miR-205-induced increase of ALP activity and osteocalcin secretion. These results confirm that miR-205 inhibits osteogenic differentiation by negatively regulating Runx2 and Smad1.

In summary, the present study showed that miR-205 acts as a negative regulator of osteogenic differentiation of VSMCs at least in part by targeting Runx2 and Smad1 and confirmed the involvement of miRNAs in vascular calcification. Our results indicate that miR-205 could be a useful marker of osteogenic differentiation and the modulation of its expression could be a potential therapeutic strategy for the prevention or treatment of a variety of diseases associated with vascular calcification.

\section{References}

1 Furmanik M, Shanahan CM: ER stress in vascular calcification. Heart 2013;99:A101-A102.

-2 Demer LL, Tintut Y: Vascular calcification: pathobiology of a multifaceted disease. Circulation 2008;117:2938-2948.

3 Nakano-Kurimoto R, Ikeda K, Uraoka M, Nakagawa Y, Yutaka K, Koide M, Takahashi T, Matoba S, Yamada H, Okigaki M, Matsubara H: Replicative senescence of vascular smooth muscle cells enhances the calcification through initiating the osteoblastic transition. Am J Physiol Heart Circ Physiol 2009;297:H1673-H1684.

-4 Dhore CR, Cleutjens JP, Lutgens E, Cleutjens KB, Geusens PP, Kitslaar PJ, Tordoir JH, Spronk HM, Vermeer C, Daemen MJ: Differential expression of bone matrix regulatory proteins in human atherosclerotic plaques. Arterioscler Thromb Vasc Biol 2001;21:1998-2003.

-5 Engelse MA, Neele JM, Bronckers AL, Pannekoek H, de Vries CJ: Vascular calcification: expression patterns of the osteoblast-specific gene core binding factor alpha- 1 and the protective factor matrix gla protein in human atherogenesis. Cardiovasc Res 2001;52:281-289.

6 Tyson KL, Reynolds JL, McNair R, Zhang Q, Weissberg PL, Shanahan CM: Osteo/chondrocytic transcription factors and their target genes exhibit distinct patterns of expression in human arterial calcification. Arterioscler Thromb Vasc Biol 2003;23:489-494.

7 Shanahan CM: Inflammation ushers in calcification: A cycle of damage and protection. Circulation 2007;116:2782-2785.

-8 Guo H, Ingolia NT, Weissman JS, Bartel DP: Mammalian microRNAs predominantly act to decrease target mRNA levels. Nature 2010;466:835-840.

-9 Huntzinger E, Izaurralde E: Gene silencing by microRNAs: contributions of translational repression and mRNA decay. Nat Rev Genet 2011;12:99-110.

10 Rana TM: Illuminating the silence: understanding the structure and function of small RNAs. Nat Rev Mol Cell Biol 2007;8:23-36.

11 Zhang C: MicroRNAs: role in cardiovascular biology and disease. Clin Sci (Lond) 2008;114:699-706.

12 Latronico MV, Catalucci D, Condorelli G: Emerging role of microRNAs in cardiovascular biology. Circ Rex 2007;101:1225-1236.

13 Goettsch C, Rauner M, Pacyna N, Hempel U, Bornstein SR, Hofbauer LC: miR-125b regulates calcification of vascular smooth muscle cells. Am J Pathol 2011;179:1594-1600. 


\section{Cellular Physiology $\quad$ Cell Physiol Biochem 2014;33:1945-1953 and Biochemistry \\ Qiao/Chen/Zhang: miR-205 Regulates Calcification of VSMCs}

-14 Cui RR, Li SJ, Liu LJ, Yi L, Liang QH, Zhu X, Liu GY, Liu Y, Wu SS, Liao XB, Yuan LQ, Mao DA, Liao EY: MicroRNA-204 regulates vascular smooth muscle cell calcification in vitro and in vivo. Cardiovasc Res 2012;96:320-329.

15 Du Y, Gao C, Liu Z, Wang L, Liu B, He F, Zhang T, Wang Y, Wang X, Xu M, Luo GZ, Zhu Y, Xu Q, Wang X, Kong W: Upregulation of a disintegrin and metalloproteinase with thrombospondin motifs-7 by miR-29 repression mediates vascular smooth muscle calcification. Arterioscler Thromb Vasc Biol 2012;32:2580-2588.

-16 Balderman JA, Lee HY, Mahoney CE, Handy DE, White K, Annis S, Lebeche D, Hajjar RJ, Loscalzo J, Leopold JA: Bone morphogenetic protein-2 decreases microRNA-30b and microRNA-30c to promote vascular smooth muscle cell calcification. J Am Heart Assoc 2012;1:e003905.

-17 Liao XB, Zhang ZY, Yuan K, Liu Y, Feng X, Cui RR, Hu YR, Yuan ZS, Gu L, Li SJ, Mao DA, Lu Q Zhou XM, de Jesus Perez VA, Yuan LQ: MiR-133a modulates osteogenic differentiation of vascular smooth muscle cells. Endocrinology 2013;154:3344-3352.

-18 Zhang Y, Xie RL, Croce CM, Stein JL, Lian JB, van Wijnen AJ, Stein GS: A program of microRNAs controls osteogenic lineage progression by targeting transcription factor Runx2. Proc Natl Acad Sci USA 2011;108:9863-9868.

19 Zhang Y, Xie RL, Gordon J, LeBlanc K, Stein JL, Lian JB, van Wijnen AJ, Stein GS: Control of mesenchymal lineage progression by microRNAs targeting skeletal gene regulators Trps1 and Runx2. J Biol Chem 2012;287:21926-21935.

20 Greene SB, Gunaratne PH, Hammond SM, Rosen JM: A putative role for microRNA-205 in mammary epithelial cell progenitors. J Cell Sci 2010;123:606-618.

21 Chen G, Deng C, Li YP: TGF-b and BMP signaling in osteoblast differentiation and bone formation. Int J Biol Sci 2012;8:272-288.

22 Tabruyn SP, Hansen S, Ojeda-Fernández ML, Bovy N, Zarrabeitia R, Recio-Poveda L, Bernabéu C, Martial JA, Botella LM, Struman I: MiR-205 is downregulated in hereditary hemorrhagic telangiectasia and impairs TGF-beta signaling pathways in endothelial cells. Angiogenesis 2013;16:877-887.

23 Hruska KA: Vascular Smooth Muscle Cells in the Pathogenesis of Vascular Calcification. Circ Res 2009;104:710-711.

24 Kovacic JC, Moreno P, Nabel EG, Hachinski V, Fuster V: Cellular senescence, vascular disease, and aging: part 2 of a 2-part review: clinical vascular disease in the elderly. Circulation 2011;123:1900-1910.

25 Arad Y, Goodman KJ, Roth M, Newstein D, Guerci AD: Coronary calcification, coronary disease risk factors, C-reactive protein, and atherosclerotic cardiovascular disease events: the St. Francis Heart Study. J Am Coll Cardiol 2005;46:158-165.

26 Wilson PW, Kauppila LI, O'Donnell CJ, Kiel DP, Hannan M, Polak JM, Cupples LA: Abdominal aortic calcific deposits are an important predictor of vascular morbidity and mortality. Circulation 2001;103:1529-1534.

27 Li B: Bone morphogenetic protein-Smad pathway as drug targets for osteoporosis and cancer therapy. Endocr Metab Immune Disord Drug Targets 2008;8:208-219.

28 Lee KS, Kim HJ, Li QL, Chi XZ, Ueta C, Komori T, Wozney JM, Kim EG, Choi JY, Ryoo HM, Bae SC: Runx2 is a common target of transforming growth factor beta1 and bone morphogenetic protein 2, and cooperation between Runx2 and Smad5 induces osteoblast-specific gene expression in the pluripotent mesenchymal precursor cell line C2C12. Mol Cell Biol 2000;20:8783-8792.

29 Jonason JH, Xiao G, Zhang M, Xing L, Chen D: Post-translational regulation of Runx2 in bone and cartilage. J Dent Res 2009;88:693-703.

30 Lin EA, Kong L, Bai XH, Luan Y, Liu CJ: miR-199a, a bone morphogenic protein 2-responsive MicroRNA, regulates chondrogenesis via direct targeting to Smad1. J Biol Chem 2009;284:11326-11335.

-31 Yin Q, Wang X, Fewell C, Cameron J, Zhu H, Baddoo M, Lin Z, Flemington EK: MicroRNA miR-155 inhibits bone morphogenetic protein (BMP) signaling and BMP-mediated Epstein-Barr virus reactivation. J Virol 2010;84:6318-6327. 